PROCEEDINGS OF THE

AMERICAN MATHEMATICAL SOCIETY

Volume 130, Number 1, Pages 183-192

S 0002-9939(01)06139-1

Article electronically published on June 8, 2001

\title{
INEQUALITIES FOR THE GAMMA FUNCTION AND ESTIMATES FOR THE VOLUME OF SECTIONS OF $B_{p}^{n}$
}

\author{
JESÚS BASTERO, FERNANDO GALVE, ANA PEÑA, AND MIGUEL ROMANCE
}

(Communicated by N. Tomczak-Jaegermann)

\begin{abstract}
Let $B_{p}^{n}=\left\{\left(x_{i}\right) \in \mathbb{R}^{n} ; \sum_{1}^{n}\left|x_{i}\right|^{p} \leq 1\right\}$ and let $E$ be a $k$-dimensional subspace of $\mathbb{R}^{n}$. We prove that $\left|E \cap B_{p}^{n}\right|_{k}^{1 / k} \geq\left|B_{p}^{n}\right|_{n}^{1 / n}$, for $1 \leq k \leq(n-1) / 2$ and $k=n-1$ whenever $1<p<2$. We also consider $0<p<1$ and other related cases. We obtain sharp inequalities involving Gamma function in order to get these results.
\end{abstract}

\section{INTRODUCTION}

In [13, J. D. Vaaler proved that all $k$-dimensional sections of the unit cube $[-1 / 2,1 / 2]^{n}$ in $\mathbb{R}^{n}$ have $k$-dimensional volume bigger than or equal to 1 . If we write $B_{p}^{n}=\left\{x \in \mathbb{R}^{n} ;\left|x_{1}\right|^{p}+\cdots+\left|x_{n}\right|^{p} \leq 1\right\}, 0<p<\infty$, M. Meyer and A. Pajor extended Vaaler's result to the range $p \in\{1\} \cup[2, \infty]$ in [8. More precisely, they showed that

$$
\left|E \cap B_{p}^{n}\right|_{k}^{1 / k} \geq\left|B_{p}^{n}\right|_{n}^{1 / n},
$$

for all $E, k$-dimensional subspace of $\mathbb{R}^{n}$. K. Ball (see [1, 2]), using Brascamp and Lieb inequality, established (0.1) for the 1-dimensional sections of any ball in $\mathbb{R}^{n}$ having a multiple of the euclidean ball as the ellipsoid of maximal volume contained in it. More recently Schmuckenschläger (see [12]) estimated the volume of the $(n-1)$-dimensional sections of $B_{p}^{n}$, for $1<p<2$, but the proof of the inequality he proposed was not correct. The aim of this paper is to give a proof of the inequality appearing in [12] (see Proposition 1.2 below), to prove (0.1) for $1 \leq k \leq(n-1) / 2$ and $1<p<2$ (see Proposition 2.2) and to give the right estimate for (0.1) for $0<p<1$ (see Proposition 2.5). Moreover, we prove (0.1) for $B=B_{2}^{n} \bigoplus_{p} B_{2}^{n}, 1 \leq p \leq 2$ (see Proposition [2.4), answering a question raised to the authors by M. Meyer.

In order to do this we need to establish sharp inequalities involving the Gamma function which have their own interest. We state and prove these inequalities in section 1 and the corresponding estimates for the volume of sections are given in section 2 .

Received by the editors May 31, 2000.

2000 Mathematics Subject Classification. Primary 52A21, 33B15; Secondary 46B20.

Key words and phrases. Gamma function, inequalities, sections of convex bodies.

The first, the third and the fourth authors were supported in part by a DGES Grant (Spain).

The fourth author was also supported by an FPI Grant (Spain). 
As usual we denote by $\|x\|_{p}=\left(\sum_{1}^{n}\left|x_{i}\right|^{p}\right)^{1 / p}$, for $x=\left(x_{1} \ldots, x_{n}\right) \in \mathbb{R}^{n}$ and $0<p<\infty$. If $A \subseteq \mathbb{R}^{k},|A|_{k}$ will represent the $k$-dimensional Lebesgue measure in $\mathbb{R}^{k}$.

\section{Some inequalities for the Gamma function}

Throughout this paper we are going to use Stirling's formula, i.e.

$$
\Gamma(1+x)=x^{x} e^{-x} \sqrt{2 \pi x} \exp \mu(x) \quad(x>0),
$$

where $\mu(x)$ is a nonincreasing and nonnegative function for $x \geq 1$ defined by

$$
\mu(x)=\frac{1}{12 x}-\frac{1}{3} \int_{0}^{\infty} \frac{p_{3}(t)}{(t+x)^{3}} d t
$$

where $p_{3}(t)$ is a 1-periodic function that for $t \in[0,1]$ is defined by $p_{3}(t)=t^{3}-$ $\frac{3}{2} t^{2}+\frac{1}{2} t$ (see, for instance, [11, pg. 62). Notice that $\left|p_{3}(t)\right| \leq \frac{1}{20}$ for all $t \geq 0$.

Proposition 1.1. The following inequalities hold:

(1) $\Gamma(1+x)^{2 / x} \leq \frac{1}{6}(x+1)(x+2) \quad(x \geq 2)$,

(2) $\Gamma(1+x)^{2 / x} \leq \frac{4}{23}(x+1)(x+2) \quad(1 \leq x \leq 2)$,

(3) $\Gamma(1+x)^{2 / x} \geq \frac{2}{\pi \sqrt{23}} x \sqrt{(x+3)(x+7)} \quad(x \geq 5)$,

(4) $\Gamma(1+x)^{2 / x} \geq \frac{1}{e^{2}}(x+1)(x+2) \quad(x \geq 1)$.

Proof. We are only going to prove (1) and (3) because (2) and (4) can be shown in a similar way.

(1) It is enough to prove that for every $x \geq 2$

$$
f(x)=\log \Gamma(1+x)-\frac{x}{2} \log \left(\frac{1}{6}(x+1)(x+2)\right) \leq 0 .
$$

Let us compute $f^{\prime}$ :

$$
f^{\prime}(x)=-\frac{2-\log 6}{2}+\psi(x+1)-\frac{\log (x+1)(x+2)}{2}+\frac{1}{x+2}+\frac{1 / 2}{x+1}
$$

where $\psi(1+x)=(\log \Gamma(1+x))^{\prime}$. Now using that

$$
\psi(1+x)<\log (x+1)-\frac{1 / 2}{x+1}-\frac{1 / 12}{(x+1)^{2}}+\frac{1 / 120}{(x+1)^{4}}
$$

for $1+x>0$ (see, for instance, [3], section 541) and considering $y=\frac{1}{x+1}$ we obtain that

$$
\sup _{x \in[2,+\infty)} f^{\prime}(x) \leq \sup _{y \in(0,1 / 3]} g(y)
$$

where

$$
g(y)=-\frac{2-\log 6}{2}-\frac{1}{2} \log (y+1)+\frac{y}{y+1}-\frac{y^{2}}{12}+\frac{y^{4}}{120} .
$$

Since $g$ is concave on $(0,1 / 3]$ and $g^{\prime}(1 / 3)>0$, we get

$$
\sup _{y \in(0,1 / 3]} g(y)=g(1 / 3)<0 .
$$


Hence $f$ is a nonincreasing function on $[2,+\infty)$ and so for every $x \geq 2$

$$
\log \Gamma(1+x)-\frac{x}{2} \log \left(\frac{1}{6}(x+1)(x+2)\right) \leq f(2)=0 .
$$

(3) Consider the function $F:[5,+\infty) \longrightarrow \mathbb{R}$ defined by

$$
F(x)=\frac{4}{x} \log \Gamma(x+1)-2 \log x-\log (x+3)-\log (x+7)-\log \frac{4}{23 \pi^{2}} .
$$

We are going to show that $F(x) \geq 0$. We have

$$
\begin{aligned}
& x F(x)=4 \log \Gamma(x+1)-2 x \log x-x \log (x+3)-x \log (x+7)-x \log \frac{4}{23 \pi^{2}} \\
& \geq 4 \log \left(x^{x} e^{-x} \sqrt{2 \pi x}\right)-2 x \log x-x \log (x+3)-x \log (x+7)-x \log \frac{4}{23 \pi^{2}}=G(x) .
\end{aligned}
$$

If we denote $\beta=-\log \frac{4 e^{4}}{23 \pi^{2}}>0$, we get that

$$
\begin{aligned}
G^{\prime}(x)= & {\left[\log \left(1-\frac{3}{x+3}\right)+\frac{3}{5 x}+\frac{3}{x+3}+\frac{\beta}{2}\right] } \\
& +\left[\log \left(1-\frac{7}{x+7}\right)+\frac{7}{5 x}+\frac{7}{x+7}+\frac{\beta}{2}\right] .
\end{aligned}
$$

We have

$$
h(y)=\log (1-y)+\frac{y}{5(1-y)}+y+\frac{\beta}{2}>0
$$

whenever $y \in[0,7 / 12]$, since $h$ is concave and $h(0), h(7 / 12)>0$. It is clear that for every $x \geq 5,7 /(x+7)$ and $3 /(x+3)$ belong to the interval $[0,7 / 12]$, therefore $G^{\prime}(x)>0$ on $[5,+\infty)$. Hence we conclude that $G(x) \geq G(5)>0$ for all $x \in[5,+\infty)$, and so $F(x)>0$, for all $x \geq 5$.

Proposition 1.2. Let $1 / 2 \leq x \leq 1$ and $y \geq 2$. Then

$$
\frac{\Gamma(1+x y)^{1+\frac{2}{y}}}{\Gamma(1+(y+2) x)} \leq \frac{\Gamma(1+x)^{3}}{\Gamma(1+3 x)} \text {. }
$$

Proof. First of all note that for every $y \geq 2$, (1.1) holds for $x=1$ and $x=1 / 2$, simply using Proposition 1.1 and because

$$
\frac{\Gamma(1+y)^{1+2 / y}}{\Gamma(3+y)}=\frac{\Gamma(1+y)^{2 / y}}{(y+2)(y+1)} \leq \frac{1}{6}
$$

and

$$
\frac{\Gamma\left(1+\frac{y}{2}\right)^{1+2 / y}}{\Gamma\left(2+\frac{y}{2}\right)}=\frac{\Gamma\left(1+\frac{y}{2}\right)^{2 / y}}{1+\frac{y}{2}} \leq \frac{2}{\sqrt{23}} \sqrt{\frac{y+4}{y+2}} \leq \frac{\pi}{6} .
$$

Now we only have to prove that for every $y \geq 2$ the function $f_{y}:\left[\frac{1}{2}, 1\right] \rightarrow \mathbf{R}$ defined by $f_{y}(x)=3 \log \Gamma(1+x)-\log \Gamma(1+3 x)-\left(1+\frac{2}{y}\right) \log \Gamma(1+x y)+\log \Gamma(1+(y+2) x)$ is concave. If we compute its derivate, we obtain

$f_{y}^{\prime \prime}(x)=3 \psi^{\prime}(1+x)-9 \psi^{\prime}(1+3 x)-y(y+2) \psi^{\prime}(1+x y)+(y+2)^{2} \psi^{\prime}(1+(y+2) x)$.

Next we use that there exists a function $\theta:(0,+\infty) \longrightarrow[0,1]$ such that for every $z>0$

$$
\psi^{\prime}(z)=\frac{1}{z}+\frac{1}{2 z^{2}}+\frac{1}{6 z^{3}}-\frac{\theta(z)}{30 z^{5}}
$$


(see [3] or 4]). Then we get that

$$
\begin{aligned}
f_{y}^{\prime \prime}(x)= & \underbrace{-\frac{1}{(x+1)\left(x+\frac{1}{3}\right)}\left[1+\frac{4}{3} \cdot \frac{x+\frac{1}{2}}{(x+1)\left(x+\frac{1}{3}\right)}\right]}_{\mathbf{S} \mathbf{1}}+\underbrace{\frac{-1+9 x^{2}+12 x^{3}}{(1+x)^{3}(1+3 x)^{3}}}_{\mathbf{S} \mathbf{2}} \\
& +\underbrace{\frac{1 / y}{\left(x+\frac{1}{y}\right)\left(x+\frac{1}{y+2}\right)}\left[1+\frac{2(y+1)}{y(y+2)} \cdot \frac{x+\frac{1}{y+1}}{\left(x+\frac{1}{y}\right)\left(x+\frac{1}{y+2}\right)}\right]}_{\mathbf{S} 3}+\alpha(x, y),
\end{aligned}
$$

where

$$
\begin{aligned}
\alpha(x, y) & \leq \frac{9}{30(1+3 x)^{5}}+\frac{y(y+2)}{30(1+x y)^{5}}-\frac{y(y+2)}{6(1+x y)^{3}}+\frac{(y+2)^{2}}{6(1+(y+2) x)^{3}} \\
& \leq \frac{9}{30(1+3 x)^{5}}+\frac{y(y+2)}{30(1+x y)^{5}} .
\end{aligned}
$$

Notice that $\mathbf{S} 1$ and $\mathbf{S} 3$ can be deduced from the identity

$$
\begin{aligned}
& \frac{-k(k+2)}{(1+k x)}+\frac{-k(k+2)}{2(1+k x)^{2}}+\frac{(k+2)^{2}}{1+(k+2) x}+ \frac{(k+2)^{2}}{2(1+(k+2) x)^{2}} \\
&=\frac{\frac{1}{k}}{\left(x+\frac{1}{k}\right)\left(x+\frac{1}{k+2}\right)}\left[1+\frac{2(k+1)}{k(k+2)} \cdot \frac{x+\frac{1}{k+1}}{\left(x+\frac{1}{k}\right)\left(x+\frac{1}{k+2}\right)}\right]
\end{aligned}
$$

applied to $k=1$ and $k=y$ respectively.

Now we are going to study each summand separately:

S1: It is easy to check that

$$
\min _{x \in\left[\frac{1}{2}, 1\right]}\left(1+\frac{4}{3} \cdot \frac{x+\frac{1}{2}}{(x+1)\left(x+\frac{1}{3}\right)}\right)=\frac{7}{4} \quad(x=1) .
$$

S2: It can be shown that

$$
\begin{gathered}
\max _{x \in\left[\frac{1}{2}, 1\right]} \frac{9 x^{2}+12 x^{3}-1}{(1+x)^{2}(1+3 x)^{2}}<\max _{x \in\left[\frac{1}{2}, 1\right]} \frac{9 x^{2}+12 x^{3}}{(1+x)^{2}(1+3 x)^{2}}=\frac{21}{64} \quad(x=1) . \\
\text { S3: } \max _{x \in\left[\frac{1}{2}, 1\right]}\left(1+\frac{2(y+1)}{y(y+2)} \cdot \frac{x+\frac{1}{y+1}}{\left(x+\frac{1}{y}\right)\left(x+\frac{1}{y+2}\right)}\right)=1+\frac{4(y+3)}{(y+2)(y+4)} \quad\left(x=\frac{1}{2}\right) .
\end{gathered}
$$

Notice that since

$$
\max _{x \in\left[\frac{1}{2}, 1\right]} \frac{(x+1)\left(x+\frac{1}{3}\right)}{\left(x+\frac{1}{y}\right)\left(x+\frac{1}{y+2}\right)}=\frac{5 y}{y+4} \quad(x=1 / 2)
$$

we get that for every $1 / 2 \leq x \leq 1$ and every $y \geq 2$

$$
\begin{aligned}
\frac{1 / y}{\left(x+\frac{1}{y}\right)\left(x+\frac{1}{y+2}\right)}\left[1+\frac{2(y+1)}{y(y+2)} \cdot \frac{x+\frac{1}{y+1}}{\left(x+\frac{1}{y}\right)\left(x+\frac{1}{y+2}\right)}\right] & \leq \frac{\frac{5}{y+4} \cdot\left(1+\frac{4(y+3)}{(y+2)(y+4)}\right)}{(x+1)\left(x+\frac{1}{3}\right)} \\
& \leq \frac{55 / 36}{(x+1)(x+1 / 3)} .
\end{aligned}
$$

On the other hand

$$
\alpha(x, y) \leq \frac{9}{30(1+3 / 2)^{5}}+\frac{y(y+2)}{30\left(\frac{y+2}{2}\right)^{5}} \leq \frac{4277}{375000}(\sim 0.012) .
$$


Therefore we obtain that for each $y \geq 2$,

$$
\begin{aligned}
f_{y}^{\prime \prime}(x) & \leq \frac{-21 / 4}{(1+x)(1+3 x)}+\frac{21 / 64}{(1+x)(1+3 x)}+\frac{55 / 36}{(x+1)(x+1 / 3)}+\frac{4277}{375000} \\
& =\frac{4277}{375000}+\frac{-\frac{105}{64}+\frac{55}{36}}{(x+1)\left(x+\frac{1}{3}\right)}<0
\end{aligned}
$$

for all $1 / 2 \leq x \leq 1$

Proposition 1.3. The function $g:[1 / 2,+\infty) \times[2,+\infty) \longrightarrow(0,+\infty)$ defined by

$$
g(x, y)=\frac{y}{\Gamma(1+y)^{1 / y}} \frac{\Gamma(1+x y)^{1 / y}}{y^{x} \Gamma(1+x)}
$$

verifies:

(1) For every $y \geq 2, g(\cdot, y)$ is nonincreasing in $[1 / 2,+\infty)$.

(2) For every $x \geq 1, g(x, \cdot)$ is nonincreasing in $[2,+\infty)$ and for every $1 / 2 \leq x \leq$ $1, g(x, \cdot)$ is nondecreasing in $[2,+\infty)$.

Proof. (1) Let $y \geq 2$. By using Stirling's formula it is easy to see that

$$
h(x, y)=g(x, y) \frac{\Gamma(1+y)^{1 / y}}{y}=y^{\frac{1}{2 y}}(2 \pi x)^{\frac{1}{2 y}-\frac{1}{2}} \exp \left(\frac{\mu(x y)}{y}-\mu(x)\right) .
$$

Since $\left|p_{3}(t)\right| \leq 1 / 20$, we have

$$
\begin{aligned}
\frac{\partial(\log h)}{\partial x}(x, y) & =-\frac{y-1}{2 x y}+\frac{1}{12 x^{2}}-\frac{1}{12 x^{2} y^{2}}+\int_{0}^{+\infty}\left(\frac{p_{3}(t)}{(t+x y)^{4}}-\frac{p_{3}(t)}{(t+x)^{4}}\right) d t \\
& \leq-\frac{y-1}{2 x y}+\frac{1}{12 x^{2}}-\frac{1}{12 x^{2} y^{2}}+\frac{1}{20} \int_{0}^{+\infty}\left(\frac{1}{(t+x)^{4}}-\frac{1}{(t+x y)^{4}}\right) d t \\
& \leq \frac{1}{2 x}\left(1-\frac{1}{y}\right)\left(-1+\frac{1}{4 x}+\frac{7}{120 x^{2}}\right)<0
\end{aligned}
$$

for all $x \geq 1 / 2$ (note that this result can be extended to $x$ strictly smaller than $1 / 2$ ). Therefore $\log h(\cdot, y)$ is a nonincreasing function in $[1 / 2,+\infty)$ and so it is $g(x, y)$.

(2) Let $x \geq 1 / 2$. If we again use Stirling's expression of the Gamma function we have

$$
g(x, y)=\frac{e^{1-x} x^{x}}{\Gamma(1+x)} x^{1 / 2 y} \exp \left(\frac{1}{y}(\mu(x y)-\mu(y))\right) .
$$

Consider the function

$$
\phi(x, y)=\frac{1}{2 y} \log x+\frac{1}{y}(\mu(x y)-\mu(y))
$$

defined for $y \geq 1$ and $x \geq 1 / 2$.

$$
\phi(x, y)=\frac{1}{2 y} \log x+\frac{1}{12 y^{2}}\left(\frac{1}{x}-1\right)-\frac{1}{3 y} \int_{0}^{\infty} p_{3}(t)\left(\frac{1}{(x y+t)^{3}}-\frac{1}{(y+t)^{3}}\right) d t
$$

Then

$$
\frac{\partial^{2} \phi}{\partial x \partial y}(x, y)=-\frac{1}{2 x y^{2}}+\frac{1}{6 x^{2} y^{3}}-4 x \int_{0}^{\infty} \frac{p_{3}(t)}{(x y+t)^{5}} d t
$$


Since $\max \left\{\left|p_{3}(t)\right| ; t \geq 0\right\} \leq \frac{1}{20}$, we achieve

$$
\begin{aligned}
\frac{\partial^{2} \phi}{\partial x \partial y}(x, y) & \leq-\frac{1}{2 y^{2} x}+\frac{1}{6 x^{2} y^{3}}+\frac{1}{20 x^{3} y^{4}} \\
& \leq-\frac{1}{2 y^{2} x}\left(1-\frac{1}{6 x}-\frac{1}{40 x^{2}}\right)<0 .
\end{aligned}
$$

Hence if $x \geq 1$,

$$
\frac{\partial \phi}{\partial y}(x, y) \leq \frac{\partial \phi}{\partial y}(1, y)=0
$$

for all $y \in[2, \infty)$ and if $1 / 2 \leq x \leq 1$,

$$
0=\frac{\partial \phi}{\partial y}(1, y) \leq \frac{\partial \phi}{\partial y}(x, y)
$$

for all $y \in[2, \infty)$ and thus the result holds.

Proposition 1.4. The following inequality holds:

$$
\Gamma(1+2 x) \Gamma\left(1+\frac{x}{2}\right)^{2} \geq 2^{x} \Gamma(1+x)^{2} \Gamma\left(1+\frac{2 x-1}{2}\right)^{2 x /(2 x-1)}
$$

for all $x \geq 5 / 2$.

Proof. We apply Stirling's formula and so we only need to achieve

$$
\left(\frac{x}{x-1 / 2}\right)^{x+1 / 2} \geq \sqrt{2}((2 x-1) \pi)^{1 /(4 x-2)}
$$

and

$$
2 \mu(x)+\frac{2 x}{2 x-1} \mu\left(\frac{2 x-1}{2}\right)-\mu(2 x)-2 \mu\left(\frac{x}{2}\right) \leq 0 .
$$

The inequality (1.2) is deduced from the fact that the function

$$
F(y)=y(1+y / 2) \log \left(1+y^{-1}\right)-\frac{y}{2} \log 2-\frac{1}{2} \log (\pi y)
$$

is convex for $y>0$. In particular since $F^{\prime}(4)>0$ and $F(4)>0$ we deduce $F(y)>0$ for all $y \geq 4$ and so the inequality is true for $x \geq 5 / 2$ (consider $2 x-1=y$ ).

In order to show (1.3) we use the corresponding expansion and we have

$$
\begin{aligned}
& 2 \mu(x)+\frac{2 x}{2 x-1} \mu\left(\frac{2 x-1}{2}\right)-\mu(2 x)-2 \mu\left(\frac{x}{2}\right)=-\frac{5}{24 x}+\frac{x}{3(2 x-1)^{2}} \\
& -\frac{1}{3} \int_{0}^{\infty} p_{3}(t)\left(\frac{2}{(x+t)^{3}}+\frac{2 x}{2 x-1} \frac{1}{(t+(2 x-1) / 2)^{3}}-\frac{1}{(2 x+t)^{3}}-\frac{2}{(t+x / 2)^{3}}\right) d t \\
& =-\frac{5}{24 x}+\frac{x}{3(2 x-1)^{2}}+\frac{1}{3} \int_{0}^{\infty} p_{3}(t)\left(\frac{2}{(t+x / 2)^{3}}-\frac{2}{(x+t)^{3}}\right) d t \\
& \quad-\frac{1}{3} \int_{0}^{\infty} p_{3}(t)\left(\frac{2 x}{2 x-1} \frac{1}{(t+(2 x-1) / 2)^{3}}-\frac{1}{(2 x+t)^{3}}\right) d t \\
& \leq \frac{1}{24 x}\left(-5+\frac{23}{20 x}+\frac{8}{(2-1 / x)^{2}}+\frac{8 / 5}{(2-1 / x)^{2}(2 x-1)}\right) \leq-\frac{0.632}{24 x}<0,
\end{aligned}
$$


since the function

$$
-5+\frac{23}{20 x}+\frac{8}{(2-1 / x)^{2}}+\frac{8 / 5}{(2-1 / x)^{2}(2 x-1)}
$$

is nonincreasing for $x \geq 2$. Therefore the result follows.

\section{The volume of Central Sections \\ OF THE UNIT BALL IN $\ell_{p}^{n}, 0<p<2$}

We apply the preceding inequalities to estimate the volume of the $k$-dimensional sections of $B_{p}^{n}$, stated in the introduction.

Proposition 2.1 (see [12]). Let $n \in \mathbb{N}, n \geq 2, p \in[1,2]$ and let $E$ be any $(n-1)$ dimensional subspace in $\mathbb{R}^{n}$. Then

$$
\left|E \cap B_{p}^{n}\right|_{n-1}^{1 / n-1} \geq\left|B_{p}^{n}\right|_{n}^{1 / n}
$$

Proof. Let $E$ be a hyperplane in $\mathbb{R}^{n}$. A well-known result (see [6]) ensures that

$$
\left|B_{p}^{n} \cap E\right|_{n-1} L_{B_{p}^{n}} \geq \frac{1}{\sqrt{12}}\left|B_{p}^{n}\right|_{n}^{(n-1) / n}
$$

where $L_{B_{p}^{n}}$ (the isotropy constant) is

$$
L_{B_{p}^{n}}^{2}=\frac{\Gamma\left(1+\frac{3}{p}\right) \Gamma\left(1+\frac{n}{p}\right)^{1+2 / n}}{12 \Gamma\left(1+\frac{n+2}{p}\right) \Gamma\left(1+\frac{1}{p}\right)^{3}}
$$

(see [9]). Hence it is enough to prove that

$$
\frac{\Gamma\left(1+\frac{3}{p}\right) \Gamma\left(1+\frac{n}{p}\right)^{1+2 / n}}{\Gamma\left(1+\frac{n+2}{p}\right) \Gamma\left(1+\frac{1}{p}\right)^{3}} \leq 1
$$

for all $n \geq 2$ and all $1 \leq p \leq 2$. Notice that this follows from Proposition 1.2 .

Proposition 2.2. Let $n \in \mathbb{N}, n \geq 2, p \in[1,2]$ and let $E$ be any $k$-dimensional subspace in $\mathbb{R}^{n}$ with $1 \leq k \leq \frac{n-1}{2}$. Then

$$
\left|E \cap B_{p}^{n}\right|_{k}^{1 / k} \geq\left|B_{p}^{n}\right|_{n}^{1 / n}
$$

Proof. Acording to K. Ball's result quoted in the introduction, we only have to consider the case $n \geq 5$.

Hölder's inequality implies that

$$
\left|E \cap B_{p}^{n}\right|_{k}^{1 / k} \geq\left|E \cap n^{\frac{1}{2}-\frac{1}{p}} B_{2}^{n}\right|_{k}^{1 / k}=n^{\frac{1}{2}-\frac{1}{p}}\left|B_{2}^{k}\right|_{k}^{1 / k}
$$

(in fact $n^{1 / 2-1 / p} B_{2}^{n}$ is the ellipsoid of maximal volume contained in $B_{p}^{n}$ ). Hence it is enough to show that

$$
n^{\frac{1}{2}-\frac{1}{p}}\left|B_{2}^{k}\right|_{k}^{1 / k} \geq\left|B_{p}^{n}\right|_{n}^{1 / n}
$$

for all $1 \leq p \leq 2$ and for all $1 \leq k \leq \frac{n-1}{2}$, that is,

$$
\frac{\Gamma\left(\frac{n}{p}+1\right)^{1 / n}}{n^{1 / p} \Gamma\left(\frac{1}{p}+1\right)} \geq \frac{\Gamma\left(\frac{k}{2}+1\right)^{1 / k}}{n^{1 / 2} \Gamma\left(\frac{1}{2}+1\right)}
$$

(see for instance [10]). 
By using Proposition [1.3, for every $1 \leq p \leq 2$ we get that $g(1 / p, n) \geq g(1, n)$; therefore it is enough to prove (2.1) for $p=1$. Furthermore, since $\Gamma(1+x)$ is log-convex on $[0,+\infty)$, the function $f(x)=\Gamma(1+x)^{\frac{1}{x}}$ is a nondecreasing function on $[0,+\infty)$, so

$$
\Gamma\left(\frac{k}{2}+1\right)^{1 / k} \leq \Gamma\left(\frac{n-1}{4}+1\right)^{\frac{2}{n-1}}
$$

for all $1 \leq k \leq \frac{n-1}{2}$. On the other hand, since $\frac{n-1}{4} \geq 1$, we can use Proposition 1.1. (1) and (2), and we obtain that

$$
\frac{\Gamma\left(\frac{n-1}{4}+1\right)^{\frac{2}{n-1}}}{\Gamma\left(\frac{1}{2}+1\right)} \leq \frac{2}{\sqrt{\pi}}\left(\frac{1}{92}(n+3)(n+7)\right)^{1 / 4} .
$$

Thus, it suffices to show that

$$
\Gamma(1+n)^{4 / n} \geq \frac{4}{23 \pi^{2}} n^{2}(n+3)(n+7)
$$

for all integers $n \geq 5$ and this is a consequence of Proposition 1.1.

Remark 2.3. If we consider

$$
K=\left\{\left(x_{1}, \ldots, x_{m}\right) \in \mathbb{R}^{n} \times \cdots \times \mathbb{R}^{n} ;\left\|x_{1}\right\|_{2}^{p}+\cdots+\left\|x_{m}\right\|_{2}^{p} \leq 1\right\},
$$

with $1 \leq p \leq 2$ and $n, m \in \mathbb{N}$, and we use the same method as in Proposition [2.2, it can be shown that for every $k$-dimensional linear subspace $E$ in $\mathbb{R}^{n m}$ with $1 \leq k \leq \frac{n m-1}{2}$

$$
|E \cap K|_{k}^{1 / k} \geq|K|_{n m}^{1 / n m}
$$

for all $p \in[1,2]$ and all $n, m \in \mathbb{N}$. The only new tool we need is the inequality

$$
\frac{n^{1 / 2} \Gamma\left(1+\frac{n}{2}\right)^{1 / n}}{\Gamma\left(1+\frac{1}{2}\right) \Gamma(n+1)^{1 / n}} \geq 1 \quad(n \geq 1)
$$

which is a consequence of Proposition 1.3. Moreover we can achieve the inequality (2.3) for all $1 \leq k \leq 2 n$, when $m=2$, and this way extends the results in [8] in this case, as is shown in the following result.

Proposition 2.4. Let $1 \leq p \leq 2, n \in \mathbb{N}$ and

$$
K=\left\{\left(x_{1}, x_{2}\right) \in \mathbb{R}^{n} \times \mathbb{R}^{n} ;\left\|x_{1}\right\|_{2}^{p}+\left\|x_{2}\right\|_{2}^{p} \leq 1\right\} .
$$

Then (2.3) holds for all $k$-dimensional subspace in $\mathbb{R}^{2 n}$, with $1 \leq k \leq 2 n$.

Proof. Following the same methods as in Proposition 2.2, we only have to prove

$$
\Gamma(1+2 n) \Gamma\left(1+\frac{n}{2}\right)^{2} \geq 2^{n} \Gamma(1+n)^{2} \Gamma\left(1+\frac{2 n-1}{2}\right)^{2 n /(2 n-1)}
$$

for $n \geq 2$. The case $n \geq 3$ is Proposition 1.4 and $n=2$ can be checked directly.

Next we are going to estimate the volume of the sections through the origin for the $p$-balls $B_{p}^{n}, 0<p<1$. We should notice that Koldobsky (see [7]) studied this problem for the particular case of central hyperplane sections. He computed the volume of these sections in terms of the Fourier transform of a power of the radial function, for every $p>0$, and he applied this result to confirm the conjecture of Meyer and Pajor on the minimal volume of these particular sections of the unit $p$-balls $B_{p}^{n}, 0<p<2$. 
Proposition 2.5. Let $E$ be any $k$-dimensional subspace of $\mathbb{R}^{n}, 1 \leq k \leq n$, and let $0<p<1$. Then

$$
\left|B_{p}^{n} \cap E\right|_{k}^{1 / k} \geq \frac{e^{1-1 / p}}{\Gamma(1+1 / p) p^{1 / p}}\left|B_{p}^{n}\right|_{n}^{1 / n}
$$

and the constant

$$
\frac{e^{1-1 / p}}{\Gamma(1+1 / p) p^{1 / p}} \in(0,1)
$$

is the good order of magnitude for fixed $n$ when $p \longrightarrow 0^{+}$.

Proof. We use the results from [8]:

$$
\begin{aligned}
\left|E \cap B_{p}^{n}\right|_{k}^{1 / k} & \geq n^{1-1 / p}\left|E \cap B_{1}^{n}\right|_{k}^{1 / k} \\
& \geq n^{1-1 / p}\left|B_{1}^{n}\right|_{n}^{1 / n} \\
& =n^{1-1 / p} \frac{\Gamma(1+n / p)^{1 / n}}{(n !)^{1 / n} \Gamma(1+1 / p)}\left|B_{p}^{n}\right|_{n}^{1 / n}
\end{aligned}
$$

By Proposition 1.3

$$
n^{1-1 / p} \frac{\Gamma(1+n / p)^{1 / n}}{(n !)^{1 / n} \Gamma(1+1 / p)}
$$

is nonincreasing with $n$ and this implies the result, since

$$
\lim _{n \rightarrow \infty} n^{1-1 / p} \frac{\Gamma(1+n / p)^{1 / n}}{(n !)^{1 / n} \Gamma(1+1 / p)}=\frac{e^{1-1 / p}}{\Gamma(1+1 / p) p^{1 / p}} .
$$

Note that this value belongs to $(0,1)$. Indeed

$$
\begin{aligned}
\frac{e^{1-1 / p}}{\Gamma(1+1 / p) p^{1 / p}} & =\frac{e \sqrt{p}}{\sqrt{2 \pi}} \exp (-\mu(1 / p)) \\
& =\frac{e}{\sqrt{2 \pi}} \exp \left(\frac{1}{2} \log p-\frac{p}{12}+\frac{1}{3} \int_{0}^{\infty} \frac{p_{3}(t)}{(1 / p+t)^{3}} d t\right)
\end{aligned}
$$

and

$$
\begin{aligned}
\frac{d}{d p}\left(\frac{1}{2} \log p-\frac{p}{12}+\frac{1}{3} \int_{0}^{\infty} \frac{p_{3}(t)}{(1 / p+t)^{3}} d t\right) & =\frac{1}{2 p}-\frac{1}{12}+\frac{1}{p^{2}} \int_{0}^{\infty} \frac{p_{3}(t)}{(1 / p+t)^{4}} d t \\
& \geq \frac{1}{2 p}-\frac{1}{12}-\frac{p}{60}>0
\end{aligned}
$$

Finally we show that the result is sharp. It is easy to check that

$$
\frac{e^{1-1 / p}}{\Gamma(1+1 / p) p^{1 / p}} \sim e \sqrt{\frac{p}{2 \pi}} \quad \text { when } p \longrightarrow 0^{+}
$$

and if we consider the 1 -dimensional subspace $E_{0}=\operatorname{span}\{(1, \ldots, 1)\} \subseteq \mathbb{R}^{n}$, then it is easy to prove that

$$
\frac{\left|B_{p}^{n} \cap E_{0}\right|_{1}}{\left|B_{p}^{n}\right|_{n}^{1 / n}}=n^{1 / 2-1 / p} \frac{\Gamma(1+n / p)^{1 / n}}{\Gamma(1+1 / p)} \sim \frac{p^{\frac{1}{2}-\frac{1}{2 n}} n^{\frac{1}{2}+\frac{1}{2 n}}}{(2 \pi)^{\frac{1}{2}-\frac{1}{2 n}}} \quad\left(p \longrightarrow 0^{+}\right) .
$$


Remark 2.6. If we now consider

$$
K=\left\{\left(x_{1}, \ldots, x_{m}\right) \in \mathbb{R}^{n} \times \cdots \times \mathbb{R}^{n} ;\left\|x_{1}\right\|_{1}^{p}+\cdots+\left\|x_{m}\right\|_{1}^{p} \leq 1\right\},
$$

with $0<p \leq 1$ and $n, m \in \mathbb{N}$, and we use the same ideas as in Proposition [2.5, it can be shown that for every $k$-dimensional linear subspace $E$ in $\mathbb{R}^{n m}, 1 \leq k \leq n m$,

$$
|E \cap K|_{k}^{1 / k} \geq \frac{e^{1-1 / p}}{\Gamma(1+1 / p) p^{1 / p}}|K|_{n m}^{1 / n m}
$$

for all $p \in(0,1]$ and all $n, m \in \mathbb{N}$.

\section{REFERENCES}

1. K.M. Ball, Volumes of sections of cubes and related problems, GAFA Israel Seminar, 1987-88, Springer Verlag, LNM 1376 (1989), pp. 251-260. MR 90i:52019

2. K.M. Ball, Shadows of convex bodies, Trans. of the AMS 327 (1991), pp. 891-901. MR 92a:52011

3. G.M. Fichtenholz, Differential und Integralrechnung II, VEB Deutscher Verlag der Wissenschaften, Berlin (1964). 1966 edition MR 39:1b

4. L. Gordon, A stochastic approach to the Gamma Function, Amer. Math. Monthly 101 (1994), pp. 858-865. MR 95k:33003

5. P.J. Grabner, R. Thichy and U.T. Zimmermann, Inequalities for the gamma function with applications to permanents, Discrete Math. 154 (1996), pp. 53-62. MR 97h:33003

6. D. Hensley, Slicing convex bodies-bounds for slice area in terms of the body's covariance, Proc. Amer. Math. Soc. 79 (1980), pp. 619-625. MR 81j:52008

7. A. Koldobsky, An application of the Fourier transform to sections of star bodies, Israel J. of Math. 106 (1999), pp. 157-164. MR 99k:42011

8. M. Meyer and A. Pajor, Sections of the Unit ball of $\ell_{p}^{n}$, J. of Funct. Anal. 80 (1), (1988), pp. 109-123. MR 89h:52010

9. V. Milman and A. Pajor, Isotropic position and inertia ellipsoids and zonoids of the unit ball of a normed n-dimensional space, GAFA Israel Seminar, 1987-88, Springer Verlag, LNM 1376 (1989), pp. 64-104. MR 90g:52003

10. G. Pisier, The volume of convex bodies and Banach Geometry, Cambridge University Press, Cambridge (1989). MR 91d:52005

11. R. Remmert, Classical topics in complex function theory, Springer-Verlag, Graduate Texts in Mathematics 172 (1998). MR 98g:30002

12. M. Schmuckenschläger, Volume of intersections and sections of the unit ball of $\ell_{p}^{n}$, Proc. Amer. Math. Soc. 126 (1998), pp. 1527-1530. MR 98j:46007

13. J.D. Vaaler, A geometric inequality with applications to linear forms, Pacific J. Math. 83 (1979), 543-553. MR 81d:52007

Departamento de Matemáticas, Facultad de Ciencias, Universidad de Zaragoza, 50009 ZARAGOZA, SPAIN

E-mail address: bastero@posta.unizar.es

Departamento de Matemáticas, Facultad de Ciencias, Universidad de Zaragoza, 50009 ZARAGOZA, SPAIN

E-mail address: two@maths.univ.edu.au

Departamento de Matemáticas, Facultad de Ciencias, Universidad de Zaragoza, 50009 ZARAGOZA, SPAin

E-mail address: anap@posta.unizar.es

Departamento de Matemáticas, Facultad de Ciencias, Universidad de Zaragoza, 50009

ZARAGOZA, SPAIN

E-mail address: mromance@posta.unizar.es 